\title{
Onboard measurement method for signaling equipment on Probe Trains
}

\author{
H. Nakamura ${ }^{1}$, S. Takahashi ${ }^{1}$, T. Hiramoto ${ }^{1}$, H. Mochizuki ${ }^{1}$ \\ \& T. Mizuma ${ }^{2}$ \\ ${ }^{1}$ Department of Electronics and Computer Science, \\ Nihon University, Japan \\ ${ }^{2}$ National Traffic Safety and Environment Laboratory, Japan
}

\begin{abstract}
An automatic train protection system ATS and a track circuit is a fundamental and essential element of a railway signaling system. At first, we mention maintenance technology of ATSS. For the stable operation of AN ATS, the management of the quality factor $(Q)$ of the ground beacon is essential and the $Q$ must be kept its value within A specified level through maintenance. Our developed new measurement method is based on the current ratio between two different frequency levels of a pick-up coil current when the train passes the beacon. And it can provide the correct $Q$ data against the distance-fluctuation. Secondly we develop the onboard method of measuring track circuit. The current of track circuit which is received through a pair of onboard signal receivers is continuously recorded and analyzed. From the analyzed data signal engineers judge the necessity of maintenance of track circuits. These measuring technologies are installed onto the probe train which is one of revenue-earning train with measurement functions. A conditionbased monitoring system is realized through the result of statistic processing of monitored data which is collected from two or more probe trains.
\end{abstract}

Keywords: signaling system, ATS, track circuit, quality factor, probe train.

\section{Introduction}

Reduction of maintenance cost is an important task for railroad transportation. The railroad operators are making efforts to reduce their maintenance costs through modernizing and laborsaving of maintenance. Since measurement by instruments installed along the railroad and measurement of track conditions require a lot of 
manpower, automatic measurement by an inspection car sometimes takes place. However, inspection cars are expensive, and because they must be run on an extra schedule, there are restrictions on their running. The inspection cars owned by the railroad operator are not sufficient. To ameliorate such a situation, we are developing a probe train [1-3]. The probe train is a commercial train that uses simple sensors to automatically measure the conditions of the track and signal facilities making up the railroad system. This article introduces the measurement functions offered by the probe train, or the measurement technology of ATS (automatic train stopping system) and track circuits, among others.

\section{Probe technology for ATS}

\subsection{Problems faced by ATS maintenance and background of technical development}

If the railroad crew should overlook a stop signal indication and miss brake handling, a safety system automatically operates the brake to protect the safety of the train. Automatic train stopping systems (ATS) are examples of that kind of system. The ATS introduced on all JR lines and many other lines in Japan is a frequency shift ATS system. The frequency shift ATS uses an $L C$ coil called a beacon installed between the rails. When a train passes over the coil, the ATS receives the signal indication from the ground. Thus it is a point control ATS. If reception of information from the point control ATS becomes impossible, it is very dangerous, because it leads to a loss of function. Therefore, quality control of beacons has become an important maintenance item. For the beacons, their quality control has been handled by the $Q$-value (quality factor) of the $L C R$ series circuit, and their performance measurement has taken place with a fixed period on the field or from the train. The $Q$-value of ATS beacons placed sporadically along the railroad has to be measured one by one using a $Q$-meter. This method of measurement is exact but requires much time and manpower, so it has been considered one of the labor-saving problems of signal maintenance.

On the other hand, automatic $Q$-value measurement also takes place using an inspection car. Since the signal reception level when a train passes over the beacon is proportional to the $Q$-value, the $Q$-value can be determined by measuring the reception level by an inspection car. However, because the reception level depends not only on the $Q$-value, but also on the relative distance from the beacon to the on-board pick-up coil and the installed condition of the beacon, measurement error has been a problem.

The authors are developing probe train technology aiming at monitoring the railroad facility condition by a commercial train. For measurement of the $Q$-value of the ATS beacon by this probe train, it is indispensable to establish a measuring technique to measure the $Q$-value in the shortest possible time and with high accuracy. Section 2 of this article reports the results of our examination and experiments. 


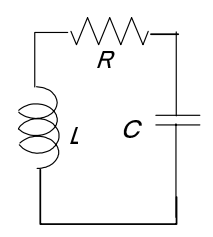

Figure 1: Equivalent circuit of ATS beacon.

\subsection{Quality control of ATS beacon}

The ATS beacon transfers information by pulling the on-board transmission frequency to the resonant frequency of the beacon. There are about 7 frequencies available nowadays, and information is obtained by discriminating the shifted frequencies through a filter on board. For this frequency shift to take place for certain, the $Q$-value of the beacon must be larger than a certain value. In maintenance work, therefore, for quality control of the beacon, the $Q$-value measurement of the beacon is performed in addition to appearance inspection by the eye.

\subsection{ATS beacon and $Q$-value}

The ATS beacon can be expressed as the $L C R$ series circuit shown in Figure 1 . The angular resonant frequency $\omega_{0}$ of the $L C R$ series circuit can be obtained from Equation (1) by setting the imaginary part of the impedance to zero.

$$
\omega_{0}^{2}=\frac{1}{L C}
$$

From the condition that the absolute values of the real part $\mathrm{R}$ and the imaginary part $\left(\omega L-(\omega C)^{-1}\right)$ are equal, the condition of Equation (2) is obtained. The cut-off angular frequencies $\omega_{b}\left(\omega_{b 1}, \omega_{b 2}\right)$ are obtained as the solutions of Equation (3).

$$
\begin{gathered}
R=\left|\omega_{b} L-\frac{1}{\omega_{b} C}\right| \\
L C \omega_{b}^{2}+R C \omega_{b}-1=0
\end{gathered}
$$

Here, letting $\omega_{b 1}<\omega_{b 2}$ and making the half width $B=f_{b 2}-f_{b 1}$, one can derive $Q=f_{0} / B$. This means that once the cut-off frequency $f_{b}$ is obtained, the $Q$-value can be calculated using the relationship with the resonant frequency. However, even if the resonant frequency of the ATS beacon is known, it is impossible to find the cut-off frequency beforehand at the on-board inspection by a probe train. Therefore, it is necessary to sweep the frequencies around a frequency in the neighborhood of the resonant frequency and determine the $Q$-value from the changes of circuit current, and this method is discussed here. In general, the flowing current 
decreases if the frequency is changed from the resonant frequency $f_{0}$. Let $I_{d}$ be the current value this time and $I_{0}$ be the current value at resonance. Then the following relationship is obtained between $Q, I_{d}$ and $I_{0}$.

$$
Q=\frac{f_{0}}{2 \Delta f} \sqrt{\left(\frac{I_{0}}{I_{d}}\right)^{2}-1}
$$

Therefore, the $Q$-value can be calculated by measuring the circuit current when the frequency is changed. Next, on-board measurement is discussed.

\subsection{The principle of $Q$-value measurement}

The discussion in the previous section holds true if the current flowing in the beacon is observable. However, the beacon current cannot be observed from on board, so it is necessary to derive a theory to measure the current through the on-board pick-up coil or a pickup as an alternative to the on-board pick-up coil. If the onboard pick-up coil system is expressed as the equivalent circuit in Figure 2(a), the circuit formed when coupled with the beacon is shown in Figure 2(b). Letting $M$ be the coefficient of mutual induction, the following simultaneous equations Equation (5) hold true.

$$
\left\{\begin{array}{l}
E_{1}=j \omega L_{1} I_{1}+j \omega M I_{2} \\
0=j \omega M I_{1}+\left(j \omega L+r+\frac{1}{j \omega C}\right) I_{2}
\end{array}\right.
$$

Solving these simultaneous equations,

$$
\frac{I_{1}}{I_{2}}=\frac{r+j\left(\omega L-\frac{1}{\omega C}\right)}{-j \omega M}
$$

Then, the current in the beacon can be calculated from the on-board current. However, $L, C$ and $M$ are constants of the beacon, so they are not observable from on board. Here, assuming that the on-board current is oscillating at the same frequency as the resonant frequency of the beacon,

$$
\frac{I_{01}}{I_{02}}=\frac{r}{-j \omega M}
$$

is obtained. On the other hand, assuming that the on-board current is oscillating at a frequency near the cut-off frequency,

$$
\frac{I_{d 1}}{I_{d 2}}=\frac{r+j\left(\omega L-\frac{1}{\omega C}\right)}{-j \omega M}=\frac{r(1+j)}{-j \omega M}
$$




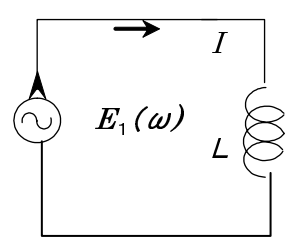

(a) onboard-pickup-coil

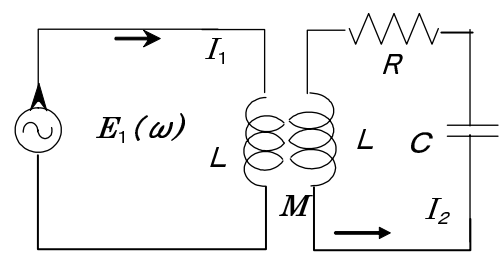

(b) coupled-circuit

Figure 2: Equivalent circuit of ATS.

The current value is found using Equations (7) and (8), and the following expression is obtained.

$$
\frac{I_{02}}{I_{d 2}}=(1+j) \frac{I_{01}}{I_{d 1}}
$$

Equation (10) can be obtained by substituting the absolute value of the expression into Equation (4).

$$
Q=\frac{f_{0}}{2 \Delta f} \sqrt{\left(\frac{I_{0}}{I_{d}}\right)^{2}-1}=\frac{f_{0}}{2 \Delta f} \sqrt{2\left(\frac{I_{01}}{I_{d 1}}\right)^{2}-1}
$$

By using Equation (10), it becomes possible to measure the $Q$-value of the ATS beacon from a probe train. That is, let $I_{01}$ be the current value when the frequency of the on-board power is made equal to the resonant frequency of the ATS beacon and $I_{d 1}$ be the current value when the frequency is varied through the half-width. Then the $Q$-value can be calculated if these current values are found. As is obvious from Equation (10), this principle shows that inspection is possible irrespective of the value of the coefficient $M$ of mutual induction between the on-board pick-up coil and beacon, or the value of $L$ of the on-board pick-up coil, so it is valuable as a radical solution to the errors and measurement variations due to the difference in height of on-board pick-up coils, which has been a problem in conventional inspection cars.

\subsection{Measurement principle verification test and results}

To verify the derived measurement principle, the authors fabricated a beacon and on-board pick-up coil on a trial basis and conducted a test according to the following procedure.

1. Fabrication of simulated beacon

The authors fabricated the coil made of an $L C R$ series circuit shown in Figure 3 as a simulated beacon, and found the impedances and resonant 


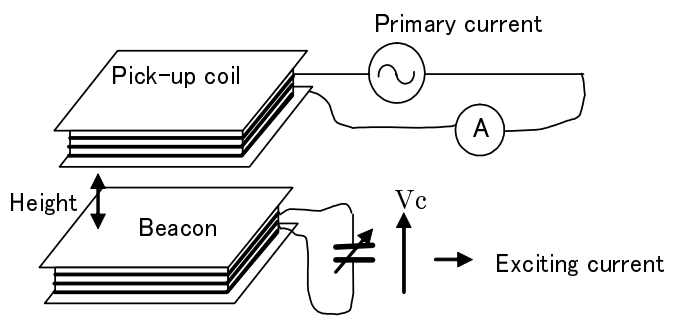

Figure 3: Test set of beacon and pick-up coil.

and cut-off frequencies for different capacitance values and calculated the $Q$-value for each.

2. Fabrication of on-board pick-up coil

The authors fabricated an pick-up coil made of the on-board coil and found the inductance and resistance values of the on-board coil.

3. Coupling test

The authors coupled the beacon and on-board pick-up coil and measured the change of primary and secondary current at that time at frequencies near the resonant and cut-off frequencies of the beacon. The same test was conducted with different coupling distances between the beacon and pick-up coil.

4. Test with different shapes of beacon

To elongate the coupling time when a train is running over, an oblong onboard pick-up coil was fabricated and a similar test was conducted using it.

5. Validation of test results

The validity of the theory was verified from the test results.

The test was conducted at various resonant frequencies and various distances between the beacon and on-board pick-up coil, and the results are shown in Figure 4. As can be seen from the test results, the developed technology is not influenced by variations of distance between the beacon and on-board pick-up coil, so it can ensure stable measurement.

\subsection{Implementation of the $Q$-value inspection system}

In the measuring method by this technology, it is necessary to excite the ATS beacon at frequencies near the resonant and cut-off frequencies of the beacon. On the other hand, the resonant frequency of a beacon differs according to the signal indications. To transmit a current wave of a conceivable resonant frequency and its cut-off frequencies to the beacon via the on-board pick-up coil, the method of sweeping the frequencies in the vicinity of the resonant frequency is conceivable. However, the time of coupling between the beacon and on-board pick-up coil is just $8 \mathrm{~ms}$ for a fast train. Thus, this method is not practical because of the severe time limit. 


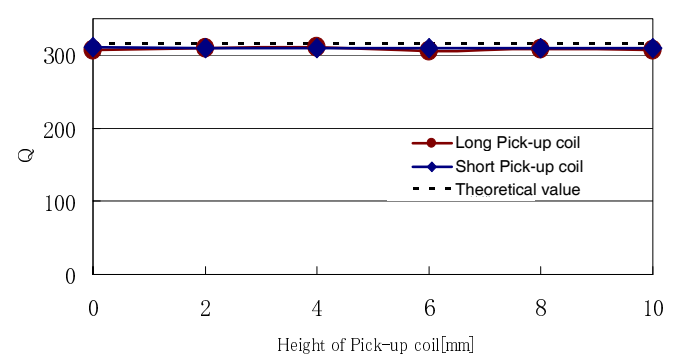

Figure 4: Effects for the alteration of pick-up coil's height.

Then, it was decided to prepare a composite probe signal containing signals of all the previously assumed frequencies and use it to excite the on-board pick-up coil. When the pick-up coil in which this composite probe signal is flowing passes over a beacon, the pick-up coil current changes. This current change is subjected to FFT analysis to extract the level of each frequency component and determine the resonance frequency of the beacon. Furthermore, the frequencies at which the level is $70 \%$ of the level of the resonant frequency are defined as cut-off frequencies, and the $Q$-value is calculated from the frequency bandwidth determined in this way.

The discussion thus far has proceeded on the assumption that the resonant frequency of the beacon is not deviated. In practice, however, the effect of deviations of the resonant frequency of the beacon must be taken into account. Thus, a test was conducted to see in what kind of error the deviations of the resonant frequency of the beacon manifest themselves.

The authors prepared a beacon having a resonant frequency deviated $0.2 \mathrm{kHz}$ with respect to the reference resonant frequency of $130 \mathrm{kHz}$ and tested it on the actual field. The half width for the resonant frequency is $0.6 \mathrm{kHz}$. The measurement results are shown in Figure 5. The resonant frequency of $130.2 \mathrm{kHz}$ of the beacon was correctly recognized from on board. Cut-off frequencies were estimated from this and measurements were made. It was found that the error would fall within the range of several percent. If, on the other hand, the deviation of the resonant frequency is not recognized and a solution is derived without correction, the error amounts to about $10 \%$, or reduces to the conventional level. For accurate implementation, therefore, it is important to prepare a composite probe signal comprising many frequency components.

For existing ATS beacons, the time of coupling with the on-board pick-up coil is about $8 \mathrm{~ms}$ if the train is running at high speed. Therefore, even if a composite probe signal comprising many frequency components is prepared, a fast real-time spectrum analyzer is needed to observe the level of each frequency component within $8 \mathrm{~ms}$. This is not suitable as a probe train facility in view of cost and space. Thus, it was decided only to record the data once in the time domain while a train is passing over the beacon, using the on-board location fixing function. This recorded data is analyzed off-line to obtain the $Q$-value of each beacon. 


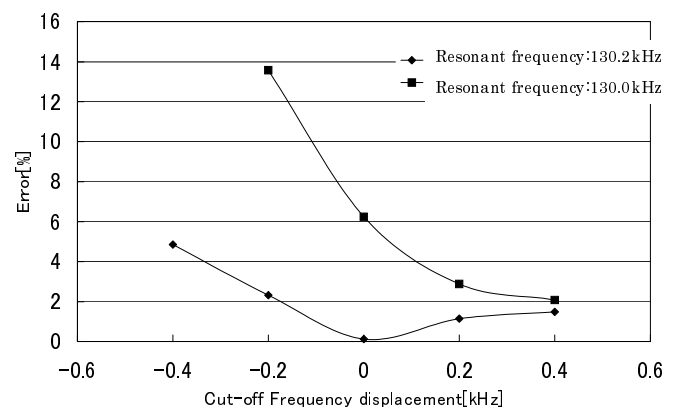

Figure 5: Measurement results of cut-off frequency displacement.

\section{Track circuit measurement technology of probe train}

Among signaling devices, the track circuit is a device to detect a train and an important element of the signaling equipment. The two-element three-position track circuit is a kind of track circuit and performs train detection using a track relay, which operates according to the relationship between the level and phase of the track circuit signal at the signal receiving terminal. The level and phase change if the track condition changes; that is, without appropriate maintenance, the track relay may detect a train erroneously although there is no train on the track. In such cases, train service becomes impossible. To prevent such a situation, periodic field maintenance takes place; however, it requires a large maintenance staff. As a measure, the field devices are placed in a centralized manner in a machine room in the station yard, and they are controlled there. However, this method incurs construction costs, so it is now restricted to the prime lines.

For measurement of the track circuit, the probe train implements automatic measurement management of the level and phase of the track circuit. For this purpose, a signal receiver is installed on board to take in the track circuit signal. In addition, an on-board signal processor finds the phase difference between the reference phase at the signal receiving terminal of the track circuit and the transmission terminal phase at the transmission terminal, and this phase difference is used as maintenance information for the track circuit. The method of measuring the track circuit from the probe train is described below.

\subsection{Track circuit reception level measurement techniques}

Reception level management of the track circuit is regarded as an important measurement item for maintenance. In track circuit measurement from the probe train, the track circuit reception level is measured by the on-board signal receiver, and the trend of level changes is used as maintenance data. In track circuit measurement from the probe train, therefore, the train location is fixed to identify the track circuit boundary. On the other hand, the on-board reception level is also influenced 


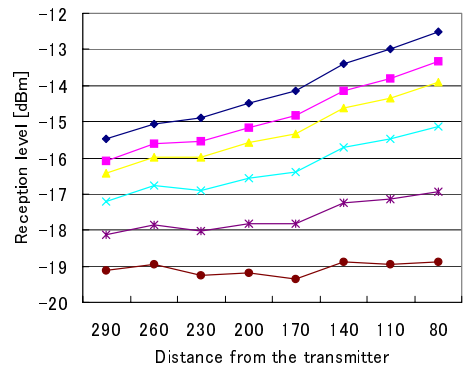

(a) $60 \mathrm{~Hz}$ track circuit

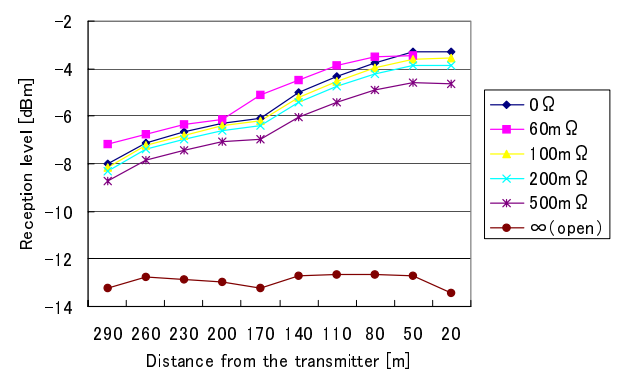

(b) $1 \mathrm{kHz}$ track circuit

Figure 6: Reception level for each short-circuit resistance at different frequencies of track circuit.

by the short-circuit resistance of the train, so its examination is indispensable.

The reception level change characteristics due to the short-circuit resistance of the train were measured for each of the frequencies of the track circuit. The results are shown in Figures 6(a) and (b). Figure 6(a) concerns a 60-Hz track circuit, and the influence of short-circuit resistance is remarkable. Therefore, if only the level at the signal receiving terminal of the track circuit is measured from on board, making some judgment based on that level alone is dangerous. This can be resolved by estimating the short-circuit resistance of the train backward from the reception level at a managed signal transmission terminal and making a correction based on that resistance. On the other hand, Figure 6(b) shows the results of measurement performed on a $1-\mathrm{kHz}$ AF(Audio Frequency) track circuit. The short-circuit resistance of the train has little influence, so there is no problem if the measurement values are used without correction.

\subsection{Track circuit phase measurement techniques}

In maintenance of the track circuit, phase measurement is also an important item along with reception level measurement. However, it has been considered difficult to inspect phase from a single received signal. The authors take the phase of the track circuit signal received at the receiving terminal when the probe enters the section of the track circuit as the reference phase and measure the phase when the probe train proceeds and reaches the signal transmission terminal of the track circuit (transmission terminal phase). The difference of these phases is used as maintenance data. Therefore, the probe train performs location fixing, that is, it knows the entry to the section of a track circuit for certain from information about the boundary points of track circuits.

In an actual track circuit, elements other than the rails which affect the phase are incorporated, so phase management must be done including these elements. This means that the mere detection of phase changes on the track circuit signal received 
by the on-board signal receiver is not sufficient. On the other hand, the technique proposed here assumes that when a track circuit is installed, phase adjustment should be complete and the optimum condition should be attained. Taking the phase difference this time as the reference, it is planned to attain the objective by managing the changes of phase difference due to aging deterioration.

\section{Conclusion}

The authors are developing a probe train, and this article introduces the measurement technology, mainly about the track circuit and ATS technology, of the signal facilities on board the train. This study is promoted as a part of the fundamental research promotion scheme "Fundamental Study on Renovation of Track Traffic System by Introduction of Probe Train Technology (Study on Signal Facility Assessment Technology by Probe Train and Study on Data Gathering Techniques)" in the transportation sector by the Japan Railway Construction, Transport and Technology Agency, an independent administrative agency.

Among the ATS systems which occupy an important position as railway safety equipment, the frequency shift ATS is greatly used, irrespective of whether on a JR or private railroad. In this study, a method using changes of the on-board pick-up coil current was examined as an inspection method independent of the coupling coefficient or geometrical positional relationship between the beacon and pickup coil, and the feasibility of this method has been confirmed. For data gathering for track circuit maintenance and measurement, it was decided to measure the level at the transmission terminal of the track circuit and the signal level at the receiving terminal and changes of phase between these terminals. As a preliminary examination, a field test was conducted to know the influence of the short-circuit resistance of the train. The conclusion obtained is that the influence is larger in the low frequency region than in the high frequency region. It is planned to verify the effectiveness of these techniques by a test using an actual train in 2006 and incorporate them as inspection and measurement functions of the probe train.

\section{References}

[1] Tsunashima, H. \& et al., Adaptive estimation of vehicle and road state using interacting multiple model approach. Proc. of the $9^{\text {th }}$ Int. Mini Conf. On Vehicle System Dynamics, Identification and Anomalies, Budapest, 2004.

[2] Tsunashima, H. \& et al., Dynamics of actively steered automated guideway transit vehicle. Proc. of the $10^{\text {th }}$ Int. Conf. On Automated People Movers, Orlando, 2005.

[3] Tsunashima, H. \& et al., Fault detection of railway track by multi-resolution analysis. Proc. of the $4^{\text {th }}$ Int. Conf. On Wavelet Analysis and Its Applications, Macau, 2005. 